\title{
Opportunistic Network Coding for Real-Time Transmission over Wireless Networks
}

\author{
Moad Y. Mowafi and Fahed H. Awad \\ Dept. of Network Engineering and Security, Jordan University of Science and \\ Technology \\ Irbid, 22110, Jordan \\ Tel: 962-2-720-1000Ｅ-mail: \{mowafi,fhawad\}@just.edu.jo
}

Mohammed A. Al-Batati

Dept. of Computer Engineering, Jordan University of Science and Technology

Irbid, 22110, Jordan

Tel: 962-2-720-1000Ｅ-mail: maalbatati082@cit.just.edu.jo

Received: August 27, 2012

DOI: 10.5296/npa.v5i1.2303
Accepted: Feb 18, 2013

Published: March 31, 2013

URL: http://dx.doi.org/10.5296/ npa.v5i1.2303

\begin{abstract}
Network coding has shown a good potential for improving network throughput and, recently, has been used in multimedia transmission over wireless networks. In addition to guaranteed throughput, real-time multimedia transmission requires bounded delay, delay variation, and packet loss. This paper presents RTOC, a new XOR-based opportunistic network coding architecture for real-time data transmission. RTOC is an application-independent architecture that takes into consideration the characteristics of real-time traffic and provides an efficient framework to optimize multimedia application requirements such as bandwidth, delay, delay variation, and loss. The performance of RTOC was evaluated using simulated video streaming traffic. The results demonstrated that RTOC can improve the real-time performance metrics such as the packet delivery ratio, end-to-end delay, jitter, and throughput by considerable margins.
\end{abstract}

Keywords: Multimedia, network coding, opportunistic coding, performance evaluation, real-time, wireless networks 


\section{Introduction}

With the growing popularity of wireless personal devices and services and the increasing demand for real-time multimedia applications, such as video streaming and videoconferencing, there is a pressing need to support various multimedia services over the wireless medium, simultaneously. However, multimedia transfer has stringent quality-of-service constraints on packet delivery time, throughput, packet order, and inter-packet delay. For example, if a packet is not received within an acceptable delay (usually milliseconds), the packet is dropped rather than being retransmitted [1]. Furthermore, in wireless networks, such traffic-intense applications would overwhelm the limited resources of the wireless nodes and degrade the quality of the received media due to the increasing demand for bandwidth and the large number of the packet collisions.

Many techniques, in different layers of the network stack, were developed for the purpose of improving the performance of multimedia transfer. For example, in some application layer protocols, multimedia traffic is compressed before transmission in order to save both storage and bandwidth. In addition, some transport layer protocols support multimedia transfer, but with limitations. For instance, the User Datagram Protocol (UDP) [2], which is an Internet standard protocol, is simple and efficient, but it does not guarantee the delivery of the packets; causing reliability problems. Other protocols, such as the Real-time Transport Protocol (RTP), and Real-time Transport Control Protocol (RTCP) [3], were built on top of UDP in order to make it more reliable.

One of the recent techniques that has been used to improve the network performance is network coding [4]. The advent of network coding has reformed the way traffic is routed in wired and wireless networks. By allowing relay nodes to combine incoming independent information flows, network coding can improve the overall network capacity and allow more traffic flows to be delivered without severely impacting the overall performance of the network. With network coding, instead of treating each packet independently, a number of packets are encoded and sent as one packet. Thus, the node's resources are efficiently utilized, more packets are delivered, and, as a result, the overall network capacity and throughput are improved.

Network coding demonstrated a good potential for improving the performance of wireless networks as well as wired networks [5]. For wireless networks, the advantage of network coding can be illustrated by the example in Fig. 1. In this example, each of Alice and Bob has a packet to send to each other. Since each is outside the communication range of the other, the transmission has to go through the router. This requires a total of four transmissions. However, by using network coding, the router can encode the two packets (e.g.; by XORing them) and broadcast the encoded packet to both, reducing the number of the needed transmissions to three, and hence achieving a coding gain of $3 / 4$.

Due to its efficiency in maximizing the total data flow [6] and reducing the energy costs [7], network coding has been recently used with different types of wireless networks and for several applications such as residential wireless mesh networks [8], many-to-many broadcast in ad hoc networks [9]-[11], and data gathering in sensor networks [12]. 


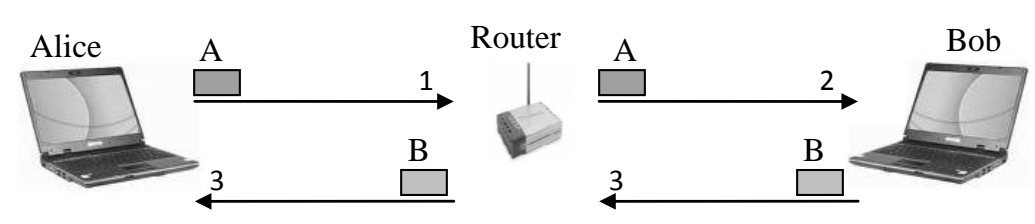

(a) Normal case

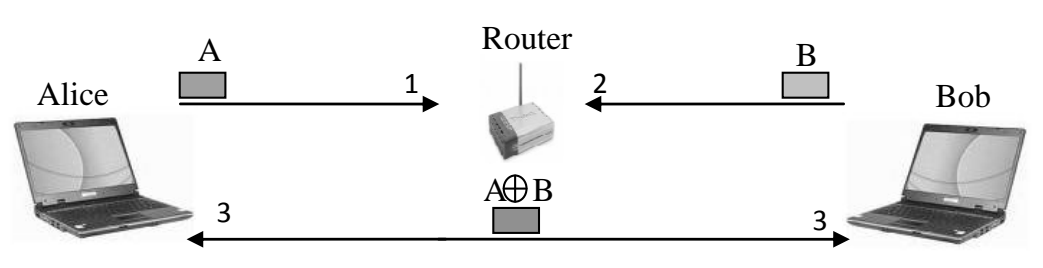

(b) Network Coding

Figure 1. Transmitting two packets in wireless networks (a) Normal case (b) Using network coding.

Network coding techniques for wireless networks have been employed at different layers of the network stack such as the physical layer [13][14], MAC layer [15], application layer [16], and between the MAC and network layers [17][18]. Moreover, other techniques were implemented across multiple layers such as in [19]-[21].

After network coding algorithms have demonstrated good potential for improving the overall wireless network capacity and throughput, network coding for multimedia transmission over wireless networks was studied in [22]-[28]. It is worth noting here that XOR operations have been widely used to implement network coding. The reason is that the complexity of XOR-based network coding in wireless networks is relatively low [18][29][30][31]. In addition, opportunistic network coding, which detects opportunities to encode as many packets as possible, has shown to be very effective in enhancing the overall capacity of wireless networks [32]-[34], especially for multimedia streaming [24][25][28].

Recently, several network coding schemes were proposed to improve multimedia transmission over wireless networks [22]-[28]. In [22], a network coding based scheduling scheme using Markov decision process (MDP) is proposed to optimize video quality in WLAN and WiMAX networks. [26] extended the MDP-based scheduling scheme in [22] considering erroneous feedback channels. In [23], a joint network coding and scheduling scheme using a partially observable Markov decision process (POMDP) is proposed to minimize the media distortion under unreliable wireless channels. [24][25] built on the network coding architecture presented in [8][18], and proposed an opportunistic network coding schemes to improve video quality and throughput in wireless mesh networks. [28] built on the work in [25], and proposed a packet delaying scheme to maximize video quality in wireless mesh networks. In [27], a network coding based pipelining scheduling scheme is presented to satisfy the delay requirements in real-time video conferencing in WiMAX networks.

Based on the above review, the existing network coding schemes were either designed for specific multimedia application/scenario or were built on top of existing network coding 
architectures that were not specifically designed to accommodate the requirements of real-time applications such as multimedia streaming. Hence, to the best of our knowledge, none of the existing network coding architectures was specifically designed for real-time traffic.

This paper presents RTOC (Real-Time Opportunistic Coding), a new XOR-based opportunistic network coding architecture for real-time data transmission. Unlike the existing network coding approaches for multimedia transmission over wireless networks, RTOC is an application-independent architecture that takes into consideration the characteristics of real-time traffic and hence offers an efficient framework for applying various techniques to satisfy different multimedia application requirements such as bandwidth, delay, delay variation, and loss.

The rest of the paper is organized as follows: The proposed RTOC architecture is introduced in Section 2, the performance evaluation of the proposed system is discussed in Section 3, and the conclusions are presented in Section 4.

\section{Real-Time Opportunistic Coding}

This section presents the new RTOC architecture and its design principles and implementation.

\subsection{RTOC Design Principles}

Even though the broadcast nature of the wireless channel and the resource limitations of the wireless nodes make the use of wireless networks for real-time applications a challenging task, some of these characteristics are considered as advantages when used with network coding. The broadcast nature of the wireless medium, the momentous data redundancy, and the significant data forwarding activities, are factors that altogether with network coding allow for new design principles that can improve the overall performance and efficiently utilize such limited resources.

The first system architecture for wireless network coding was presented by COPE (Coding Opportunistically) [8][18]. COPE introduced the concepts of opportunistic listening and opportunistic coding. With opportunistic listening, due to the broadcast nature of the wireless medium, the wireless node has the opportunity to overhear packets sent to other nodes, and is allowed to store the overheard packets for a limited period. With opportunistic coding, the node looks for opportunities to encode as many packets as possible, ensuring that the recipients can decode their packets. This can be illustrated by the example in Fig. 2. In this example, node B has 4 packets in its output queue P1, P2, P3 and P4. Each packet needs to be forwarded to one of node B's neighbors (A, C, and D). Based on its local information (i.e.; overheard or exchanged information), node B knows which packets each neighbor has. When node B is ready to transmit a packet, it takes the first packet from its packet queue and starts the coding operation (i.e.; searching for other packets to be encoded with the selected packet). Now, node B has multiple coding options, as shown in Fig. 2. In Case 1, node B can 


\section{Macrothink}

XOR P1 with P2 and broadcast the result. In this case, node $\mathrm{C}$ can reconstruct $\mathrm{P} 2$ since it already has $\mathrm{P} 1$ and needs $\mathrm{P} 2$, but node $\mathrm{A}$ cannot reconstruct any of them since it has neither P1 nor P2 in its buffer. In Case 2, node B can XOR P1 with P3. In this case, nodes A and C can decode their original packets, which is better than Case 1. However, the best coding decision is Case 3, where node B can XOR P1, P3, and P4. In this case, nodes A, C, and D can reconstruct their original packets.

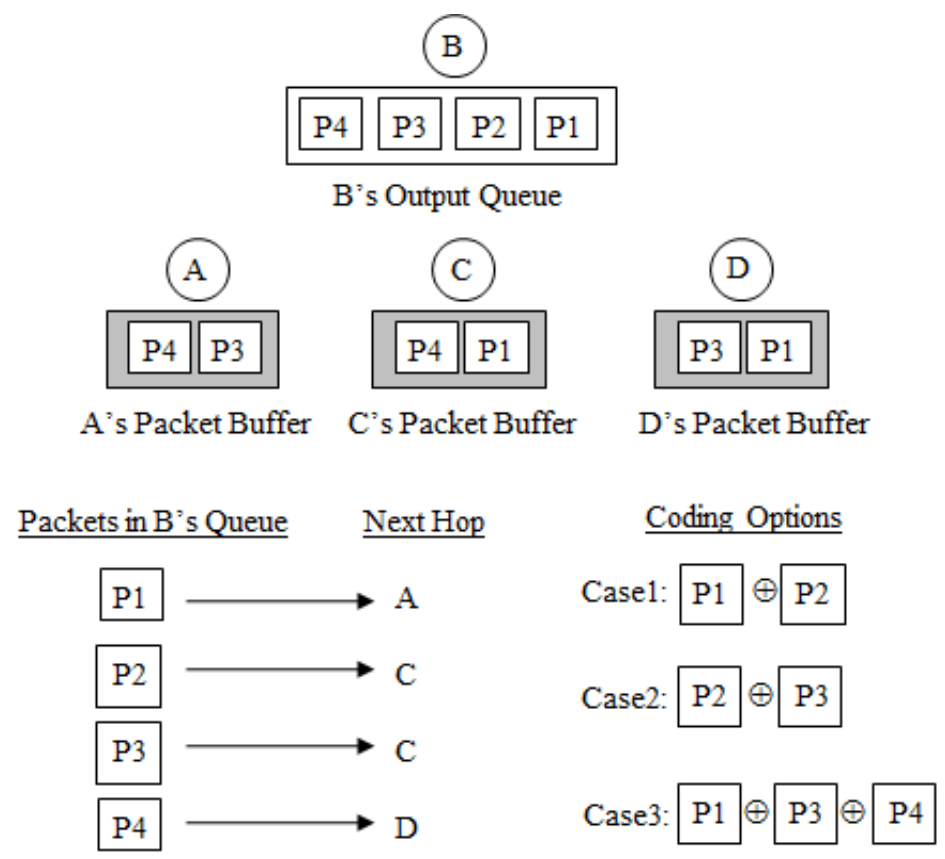

Figure 2. Example of opportunistic coding.

Even though RTOC adopts COPE's basic principles of opportunistic listening and opportunistic coding, the two systems are primarily different. COPE was designed for regular non-real-time traffic and hence the reliability of data transfer was granted higher priority than the timely delivery of data. Whereas RTOC, being specifically designed for real-time traffic, gives higher priority to the packet delivery time and inter-packet delay by minimizing the overhead associated with the encoding and decoding processes, as explained in Section 2.2. Thus, the design principles used in RTOC cope with the performance characteristics required for real-time transmission.

\subsection{RTOC Packet Coding Algorithm}

In order to reduce the overhead associated with the packet encoding and decoding processes, a simple and fast packet coding algorithm, called RTOC Packet Coding Algorithm (RPCA) was developed. RPCA uses one virtual FIFO queue for each neighbor node, where each queue contains the packets destined to that neighbor node. When a node is ready to send a packet $p$ to the next hop node $N$, it picks the head of the output queue $Q(N)$ and for each of neighbor node $i$, it checks (using the stored information about what each neighbor node has in its repository) whether encoding $p$ with the head of the virtual queue $Q(i), p_{i}$, would benefit any of the neighbor nodes. If it does, then $p_{i}$ is added to the list of native packets to be encoded. The final encoded packet is then transmitted over the wireless channel. On the other 
hand, if no encoding is found useful, then $p$ is sent without encoding. The more packets are encoded, the less the total number of transmissions and hence the less the number of packet collisions and transmission overhead. If the packets to be encoded have different sizes, the smaller packets are padded with zeros. This way, no searching for the same-length packets is necessary and no packet reordering would occur, which minimizes the packet encoding time and makes it suitable for real-time traffic. RPCA is shown in Table 1.

Table 1. RTOC Packet Coding Algorithm.

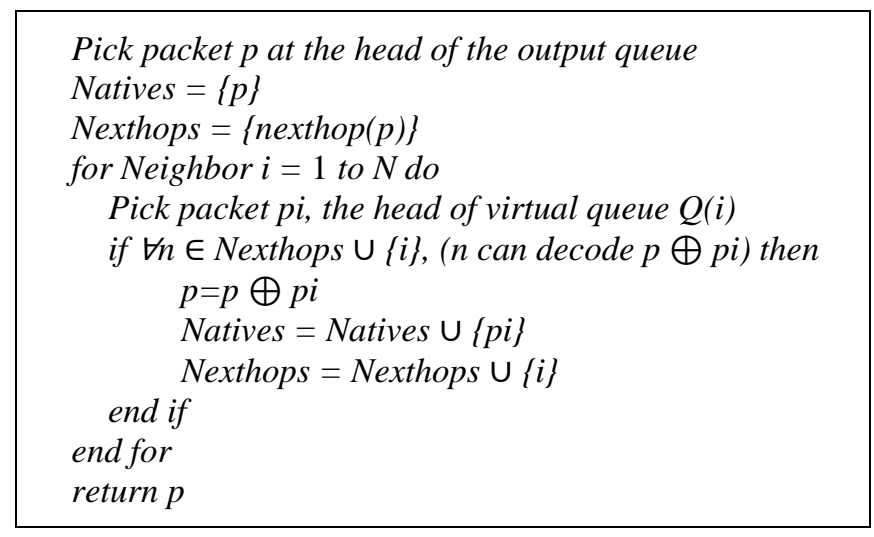

\subsection{RTOC Implementation}

RTOC was implemented above and below the MAC layer independently of the MAC protocol. The RTOC implementation consists of three components: the Packet Pool, the RTOC Encoder, and the RTOC Decoder; as shown in Fig. 3.

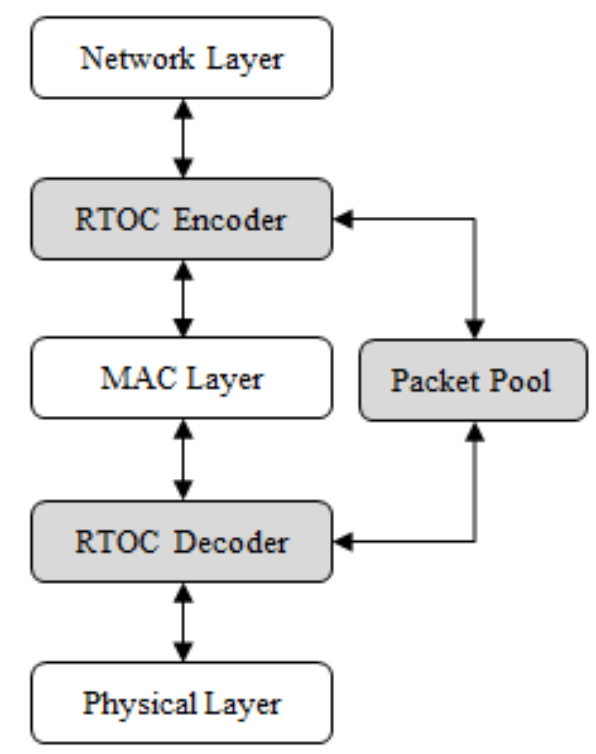

Figure 3. The RTOC implementation.

The Packet Pool is a buffer, where a node stores all packets heard, received, and sent in the past $T$ period. It provides the information needed for packet encoding and decoding. For instance, consider three nodes: Alice, Bob, and an intermediate node. For the intermediate node to encode and send packets B and A to both Alice and Bob; respectively, it needs to 
make sure that Bob has packet B and Alice has packet A. This information can be found in the buffers corresponding to Alice and Bob in the intermediate node's packet pool. If both packets are found, then the encoding is performed. At the receiver side, Bob, for example, finds, via header information, that the received packet is encoded and that the original (native) packets are A and B. Therefore, it looks up packet B from its packet pool and decodes packet A. The same thing applies to Alice.

The RTOC Encoder resides above the MAC layer. It intercepts and manages the outgoing traffic being passed to the MAC layer for transmission via the packet encoding algorithm; such as the RPCA. When the MAC layer requests a packet to be transmitted, the packet encoding algorithm searches the packet pool for other packets that can be encoded with that packet. The encoded packet is then passed to the MAC layer for transmission. In this case, in order for the receiver to obtain its original packet, it needs to know which native packets are encoded. Therefore, a new header, called RTOC_Header, is inserted between the MAC and IP headers. The RTOC_Header contains the ID of each encoded native packet, which is a 32-bit hash of the packet's source IP address and IP sequence number, followed by the MAC address of the native packet's next hop. Thus, when the encoded packet is received, the RTOC_Header is processed and the original packet is decoded with the help of the packet pool.

The RTOC Decoder resides below the MAC layer. It intercepts and manages the incoming traffic being passed to the MAC layer from below. When a packet is received and passed to the MAC layer, the RTOC decoder intercepts the encoded packet, reads the RTOC_Header, and decodes its original packet with the help of the packet pool. The packet pool is then updated with the decoded packet.

\subsection{RTOC MAC Modes}

RTOC exploits the broadcast nature of the wireless medium while sending the encoded packets from one hop to the next. The IEEE 802.11 MAC standard has two transmission modes: unicast and broadcast. The unicast mode is reliable as it requires the receiver node to send back an acknowledgment. If no acknowledgment is received within a specific time interval, the packet is retransmitted, whereas the broadcast mode is unreliable and hence does not use acknowledgments or retransmissions. Therefore, the broadcast mode incurs much less overhead than the unicast mode even though there is no guarantee that the packet is delivered. Since real-time applications are more sensitive to timely delivery of packets than the reliability or the in-order delivery of packets, the broadcast mode is generally more suited to work well with RTOC than the unicast mode as far as the packet delivery time is concerned. However, since there is no guarantee that the data are being delivered correctly, there is always a chance that the encoded packet is lost due to collision or interference. This may limit the packet delivery ratio and hence the overall throughput; due to the loss of all native packets encoded. Therefore, a quasi-broadcast MAC mode was developed in order to improve the packet delivery ratio without severely impacting the packet delivery time. Unlike the pseudo-broadcast used in COPE, which requires that all decoded packets be acknowledged via delayed acknowledgments, the RTOC quasi-broadcast requires only one 
recipient to acknowledge the sender. This provides an acceptable level of reliable data transfer with minimal overhead because, in wireless transmission, if a packet is received correctly by one node, then most probably all other nodes have also received it correctly. The following sections describe the two MAC modes used in this research in more details.

\subsubsection{The RTOC Broadcast Mode}

Fig. 4 shows the operation of RTOC with broadcast mode at both the sender and the receiver. At the sender side, the node takes the packet at the head of its output queue, and tries to encode it with the native packets in the packet pool. The node then updates the packet pool with the native packets to be sent, and adds the RTOC_Header that lists the next hops of the native packets. The encoded packet is then transmitted with the broadcast MAC address (i.e.; the destination MAC address or DMAC = FF:FF:FF:FF:FF:FF). At the receiver side, the RTOC decoder intercepts the encoded packet and, if the packet is decodable, it reconstructs the original packet and updates the packet pool. Then, if it finds that it is one of the next hops, then it passes the original packet to the MAC layer and disables the corresponding acknowledgment, since the broadcast mode does not require an acknowledgment.

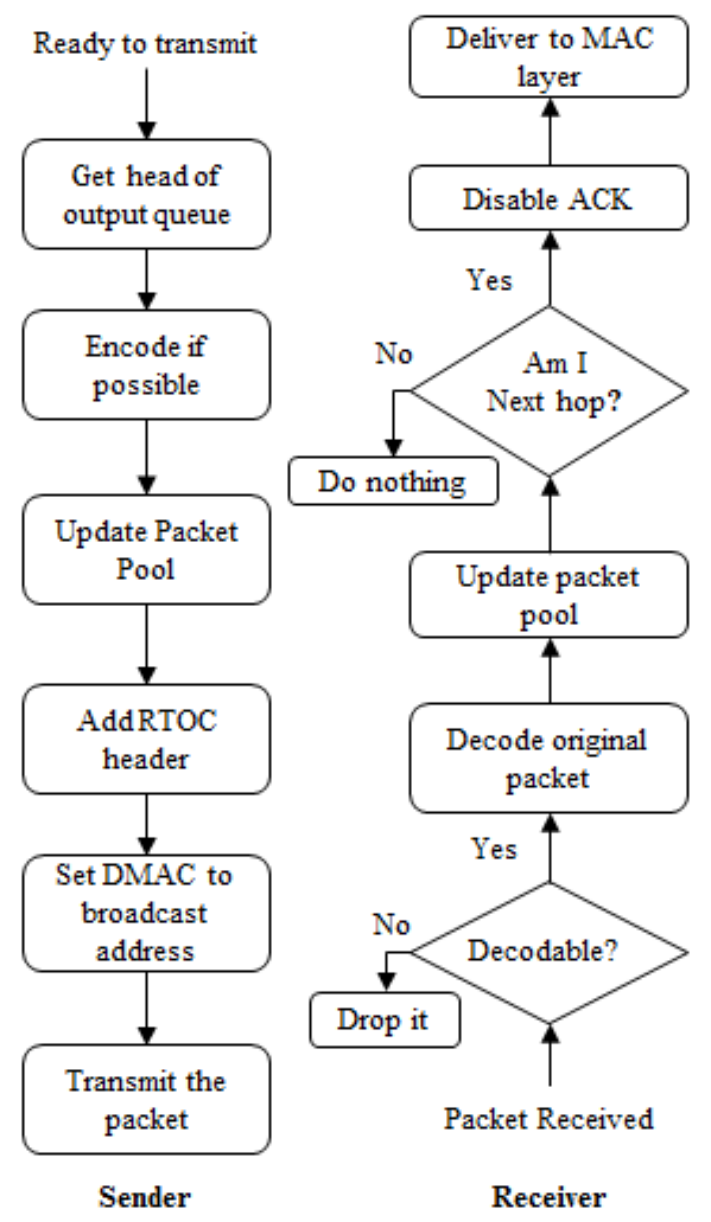

Figure 4. Flowchart for RTOC with broadcast mode.

\subsubsection{The RTOC Quasi-Broadcast Mode}

Fig. 5 shows the operation of RTOC with quasi-broadcast mode at both the sender and 


\section{Macrothink}

the receiver. At the sender side, the node takes the packet at the head of its output queue, and tries to encode it with the native packets in the packet pool. The node then updates the packet pool with the native packets to be sent, and adds the RTOC_Header that lists the next hops of the native packets. The encoded packet is then sent using the MAC address of one of the intended receivers. Since all nodes use opportunistic listening, they can overhear packets not addressed to them. At the receiver side, the RTOC decoder in each receiver node intercepts the encoded packet and, if the packet is decodable, it reconstructs the original packet and updates the packet pool. Then the node checks the RTOC_Header to see if it is one of the next hops. If the node finds that it is a next hop, it checks whether the destination MAC address matches its own MAC address. If so, it enables the corresponding acknowledgment and passes the original packet to the MAC layer. Otherwise, the corresponding acknowledgment is disabled. Thus, only one acknowledgment is sent back to the sender.

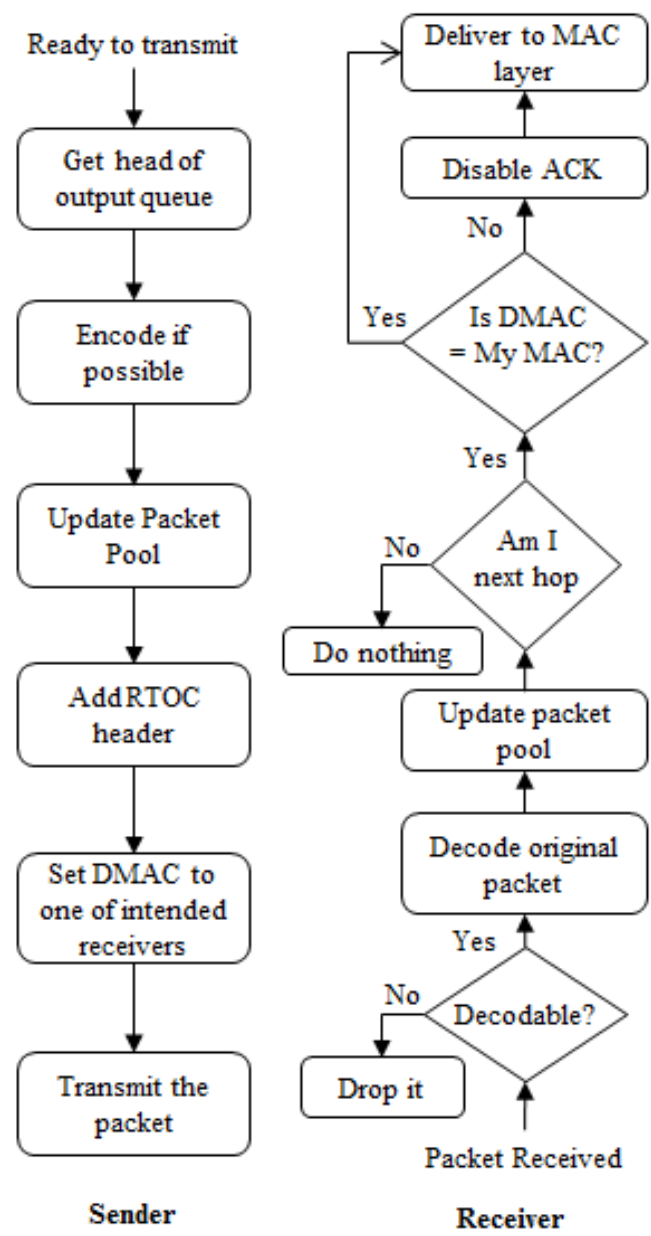

Figure 5. Flowchart for RTOC with quasi-broadcast mode.

\section{Performance Evaluation}

The performance of RTOC was evaluated via simulation, where three modes of MAC operation were implemented and tested:

1. Regular MAC operation without network coding (WNC). 
2. RTOC network coding with broadcast mode (BNC).

3. RTOC network coding with quasi-broadcast mode (QBNC).

The WNC mode was used as a reference mode for evaluating the performance of RTOC and for comparing its two MAC modes of operations: BNC and QBNC.

\subsection{Performance Metrics}

The performance metrics used to evaluate RTOC are:

1. The Packets Delivery Ratio (PDR), which is the ratio of the number of correctly received packets, $\mathrm{P}_{\mathrm{r}}$, to the number of sent packets, $\mathrm{P}_{\mathrm{s}}$. The PDR is defined as:

$$
P D R=\frac{P_{r}}{P_{s}}
$$

2. The end-to-end packet delay, which is the time it takes to transfer a packet from the sender to the receiver. This includes all the network delays in between; such as queuing, processing at intermediate nodes, and retransmission delays at the MAC layer.

3. The packet inter-arrival Jitter $(\mathrm{J})$, which is defined according to the RTP standard (RFC 3550 [3]) as the mean deviation of the difference (D) in packet spacing at the receiver compared to the sender for a pair of consecutive packets. For two consecutive packets $i$ and $j$, the difference D can be calculated as follows:

$$
D(i, j)=\left(R_{j}-R_{i}\right)-\left(S_{j}-S_{i}\right)
$$

where $R$ is the packet arrival time in RTP time-stamp units, and $S$ is the packet RTP time-stamp. Accordingly, the inter-arrival jitter for the received packet $i, \mathrm{~J}(i)$, is calculated as follows:

$$
J(i)=J(i-1)+\frac{|D(i-1, i)|-J(i-1)}{16}
$$

4. The Coding Opportunity Ratio (COR), which is the ratio of the number of coding operations performed by the network to the number of originated packets. This represents the efficiency of RTOC in utilizing the overheard packets to reduce the number of transmitted packets.

5. The throughput (TH), which is the average rate of successful data delivery per unit time over a communication channel. It is usually measured in bits per second, and can be calculated as follows:

$$
T H=\frac{P_{r} \times L}{T}
$$

where $L$ is the packet size in bits and $T$ is the total time of data transfer.

\subsection{Simulation Environment and Setup}

The performance of RTOC was evaluated using the OMNET++ discrete-event simulation environment [35] with INET network simulation framework [36] and INETMANET extension for ad hoc wireless routing protocols [37]. The RTP implementation in the INET 
framework offers transmission of simulated MPEG video over RTP. The MPEG data, which are fragmented into packets with size less than a typical LAN/WAN MTU, are encapsulated into RTP packets; as described in RFC 2550 [38]. In order to evaluate the potential of RTOC in improving the performance metrics of real-time multimedia transmission, RTOC was built for OMNET++ and was integrated with the INET framework. Then, the performance of transmission of the simulated MPEG video over RTP was evaluated with and without RTOC and the relative difference was measured. The simulation was performed with two main goals in mind:

1. Study the performance of RTOC, in terms of the number of coding opportunities.

2. Enumerate the percent improvement offered by RTOC on the performance of RTP; represented by the metrics defined earlier.

To achieve these goals, a number of stationary wireless nodes were randomly and uniformly distributed within an area, where groups of up to 3 nodes were created randomly. Each node initiates RTP sessions with the other node(s) in its group; forming active RTP sessions. This scenario was repeated several times, each with a different random node distribution, and the results were averaged out. Each of the three MAC modes, defined earlier (i.e.; BNC, QBNC and WNC), was used with every trial. In addition, each trial was repeated with three different active node ratios (ANRs): $10 \%$ (i.e.; low), $50 \%$ (i.e.; medium), and 90\% (i.e.; high), where ANR is the ratio of active nodes to the total number of nodes. However, in order to demonstrate the benefit of RTOC, the traffic load in the network was maintained at a moderate level regardless of the number of active nodes; avoiding the network traffic extremes. The reason is that RTOC relies on the broadcast nature and the corresponding data redundancy of the wireless channel; as discussed earlier. Therefore, when the network traffic is very light, the queues at the relay nodes are mostly empty and hence the encoding opportunity would be scarce and the network would behave as if there is no network coding used. On the other hand, when the network traffic is very high, all nodes would be congested and hence the packet error rate would be high. As a result, there will be no distinction on whether network coding is used or not. The simulation parameters used are listed in Table 2.

Table 2. Simulation Parameters.

\begin{tabular}{|l|l|}
\hline \multicolumn{1}{|c|}{ Parameter Type } & \multicolumn{1}{c|}{ Parameter Value } \\
\hline \hline Number of Nodes & 50 \\
\hline Node Distribution & Uniform \\
\hline Network Size & $1000 \times 1000 \mathrm{~m}^{2}$ \\
\hline Nodes Mobility & Stationary \\
\hline Active Node Ratios & $10 \%, 50 \%$, and $90 \%$ \\
\hline Transmission Range & $250 \mathrm{~m}$ \\
\hline MAC Protocol & IEEE $802.11 \mathrm{~g}$ \\
\hline Radio Frequency & $2.4 \mathrm{GHz}$ \\
\hline Channel Bandwidth & $54 \mathrm{Mbps}$ \\
\hline Wireless Routing Protocol & Dynamic Source Routing (DSR) \\
\hline
\end{tabular}

It should be emphasized here that the actual performance of the simulated application 
and the associated impact of the various network conditions and protocols used, such as the routing and the channel access algorithms, are out of scope of this research. The focus is rather on the relative performance improvement that RTOC is ought to provide.

\subsection{Simulation Results and Analysis}

In this section, the simulation results are presented and discussed for each of the performance metrics listed above.

\subsubsection{The Packet Delivery Ratio}

The PDR depends mainly on the transmission mode used (i.e.; whether reliable or unreliable) and the packet error rate caused by packet collisions. Packet collisions are caused by simultaneous transmissions on the communication channel by two or more nodes contending for channel access. Hence, as the number of active nodes on the channel increases, the probability of packet collision increases. Therefore, the use of network coding has a two-fold effect on the PDR. That is, as network coding combines more than one packet per transmission, the overall average number of transmissions per node decreases, which in turn reduces both the contention for channel access and the probability of packet collision. On the other hand, each packet collision that involves an encoded packet results in losing more than one original packet, especially if an unreliable transmission mode is used.

Fig. 6 shows the percent improvement of RTOC on PDR compared to the WNC mode. It is shown that, regardless of ANR, using QBNC mode improves the number of successfully delivered packets by up to $33 \%$; compared to WNC. On the other hand, using BNC mode slightly degrades the number of delivered packets. The reason for such difference is that QBNC is reliable since the encoded packet is acknowledged by one of the recipients, which enables the sender to recover from packet errors, whereas BNC is an unreliable mode. However, the reason that the degradation caused by BNC is not severe, compared to WNC, is because using network coding reduces the access channel contention and hence the average error rate per original packet is reduced. On the other hand, the negative impact of increasing ANR on the PDR is related to the increased channel contention and the associated packet loss and waiting overhead; as more nodes are involved in packet transmission and relaying. The reason BNC is less affected by the increased contention than QBNC is that there are no packet retransmissions or waiting overhead. 


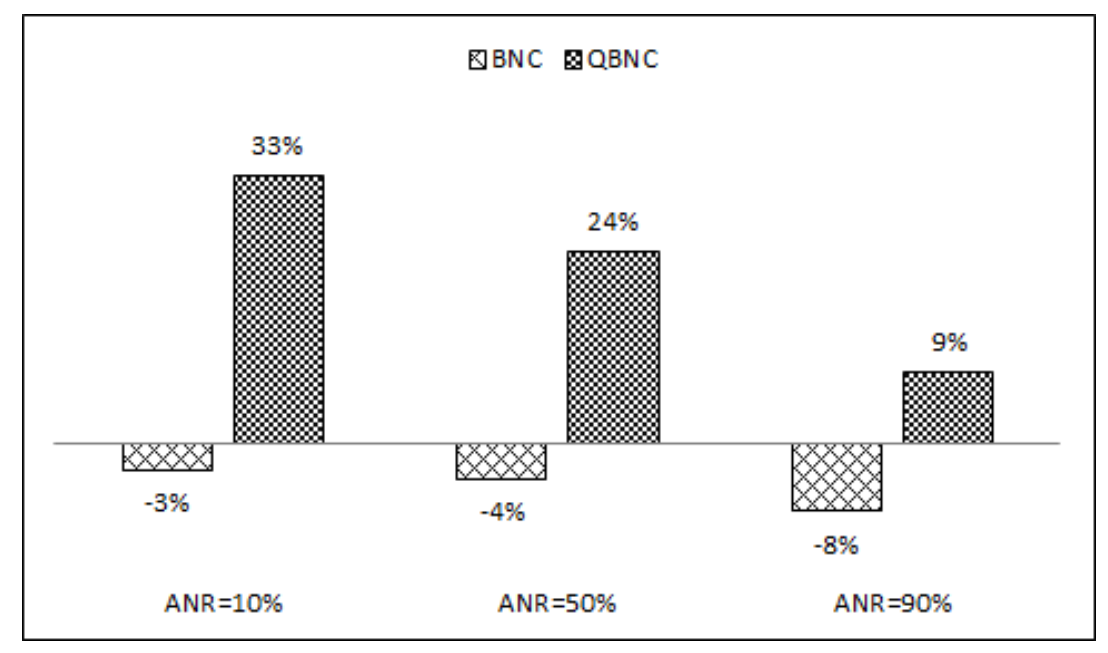

Figure 6. The RTOC improvement on the packet delivery ratio.

\subsubsection{The End-to-End Packet Delay}

The packet delivery time relies mainly on the traffic congestion and the overhead associated with the packet transmission over the communication channel. Therefore, as network coding combines packets before transmission, the overall average transmission overhead per original packet is reduced.

Fig. 7 shows the percent improvement of RTOC on the end-to-end packet delay. It is shown that, regardless of the number of active nodes, both BNC and QBNC deliver the packets much faster than WNC, which means that network coding has a significant improvement on the end-to-end delay in the general case. In addition, BNC is shown to achieve much better delay reduction than QBNC. The reason is that BNC does not have to wait for the packets to be acknowledged, which eliminates the associated overhead.

Regarding the impact of the number of active nodes on the packet delivery time, as the contention for channel access increases, as discussed earlier, the average transmission overhead at each relay node increases, which limits the benefit of using network coding. Nevertheless, this impact is barely noticeable on BNC because lost packets are not accounted for in the average end-to-end delay, while for QBNC, the packet errors are recovered via retransmission and hence the associated delivery time is extended. 


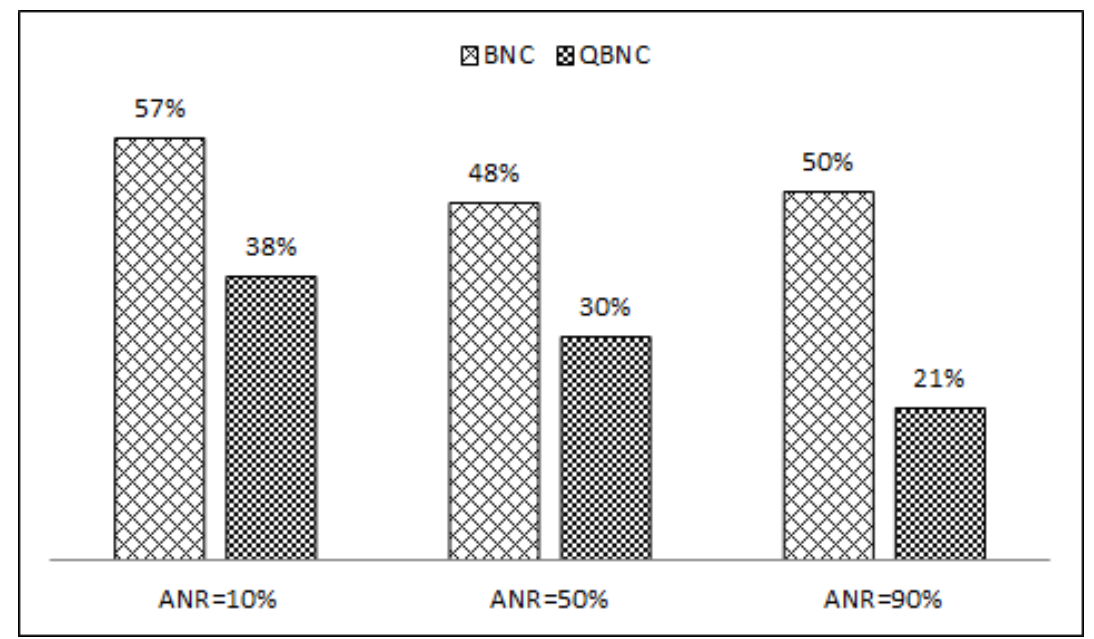

Figure 7. The RTOC improvement on the end-to-end packet delay.

\subsubsection{The Packet Inter-Arrival Jitter}

The packet inter-arrival jitter represents the deviation of the inter-packet delay at the receiver side. Therefore, as packets get delayed or lost, the jitter becomes worse. In addition, in real-time multimedia transmission, if a packet is delayed beyond a certain threshold, it gets dropped since it becomes useless, which negatively impacts the overall packet jitter.

Fig. 8 shows the RTOC improvement on the packet inter-arrival jitter. It is noticed that QBNC improves the jitter considerably at low and medium ANR, whereas at high ANR, as the packet delays and losses increase due to incremental channel access contention, the improvement on the jitter becomes very small. On the other hand, due to its associated packet losses, BNC degrades the jitter all the time.

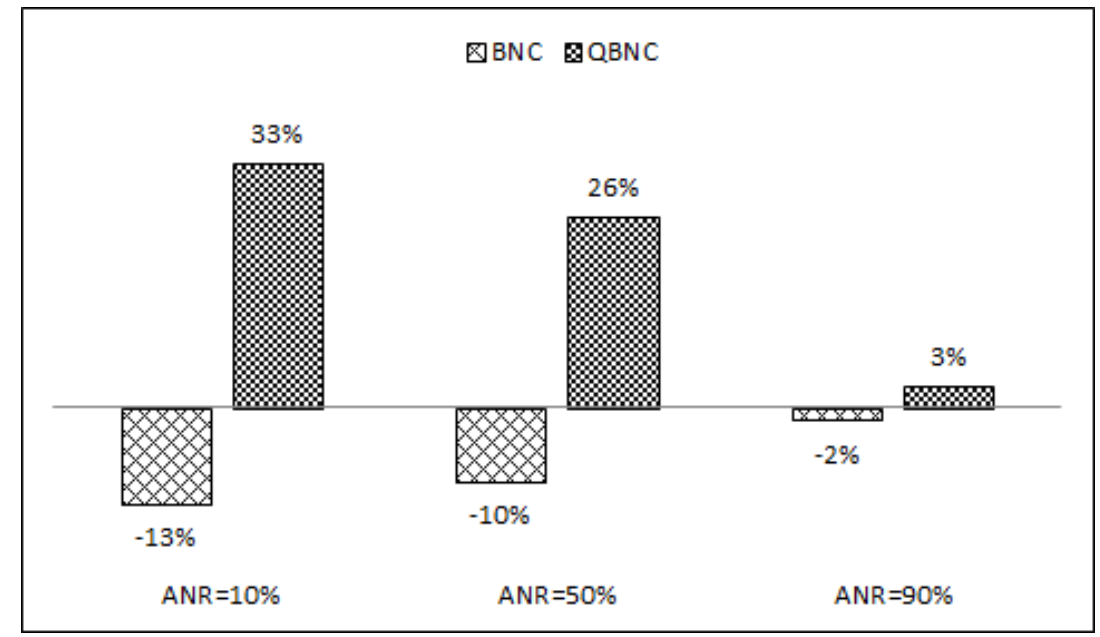

Figure 8. The RTOC improvement on the packet inter-arrival jitter.

\subsubsection{The Coding Opportunity Ratio}

The performance of RTOC relies heavily on the number of coding opportunities at the relay nodes, which is, in turn, dependent on the number of overheard packets on the channel. 
Fig. 9 shows the COR achieved by RTOC for each of the BNC and QBNC MAC modes. It shows that the QBNC mode leads to better coding opportunities than BNC. The reason is that BNC, being unreliable, loses a lot more packets than QBNC, as seen in Fig. 6, which reduces the opportunities for packet coding at the relay nodes. In addition, as the ANR increases, the COR decreases since more packets are lost; as explained earlier. Note that the behavior of the COR follows that of the PDR.

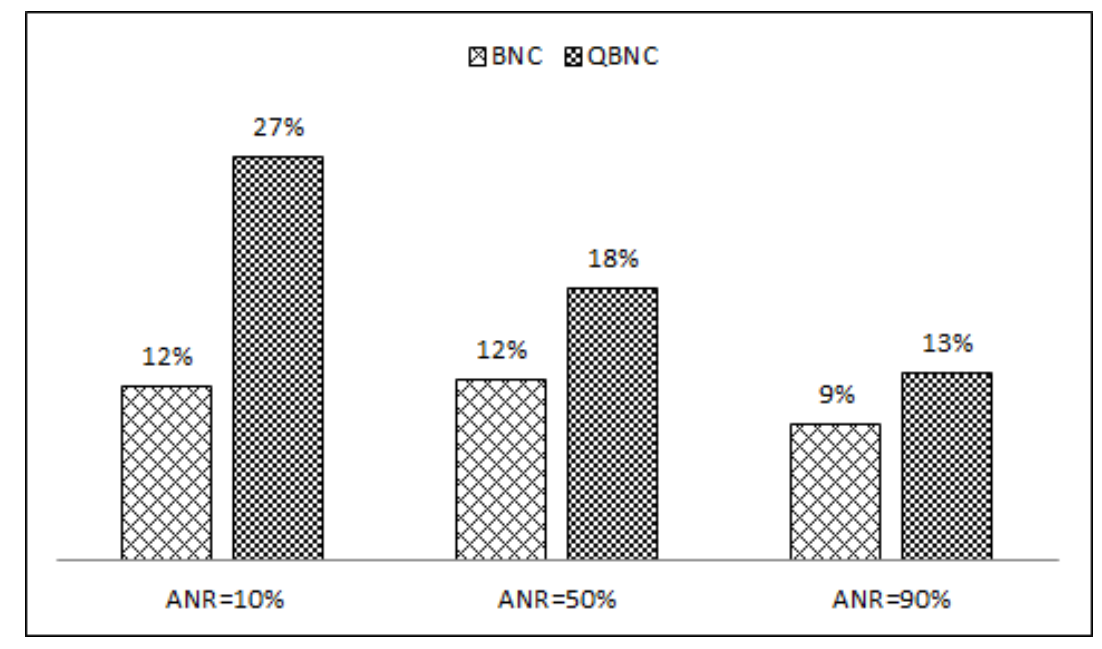

Figure 9. The ratio of the number of encoding operations to the number of sent packets.

\subsubsection{The Network Throughput}

Fig. 10 shows the improvement of RTOC on the network throughput. Since the network throughput is directly dependent on all other metrics discussed above, the impact of RTOC on it is a combination of its impact on them. Following the same trend, QBNC provides a significant improvement on the network throughput, especially at low and medium ANR, whereas BNC slightly degrades it. At high ANR, the relatively low PDR and the high jitter caused by the network contention limit the benefit of RTOC.

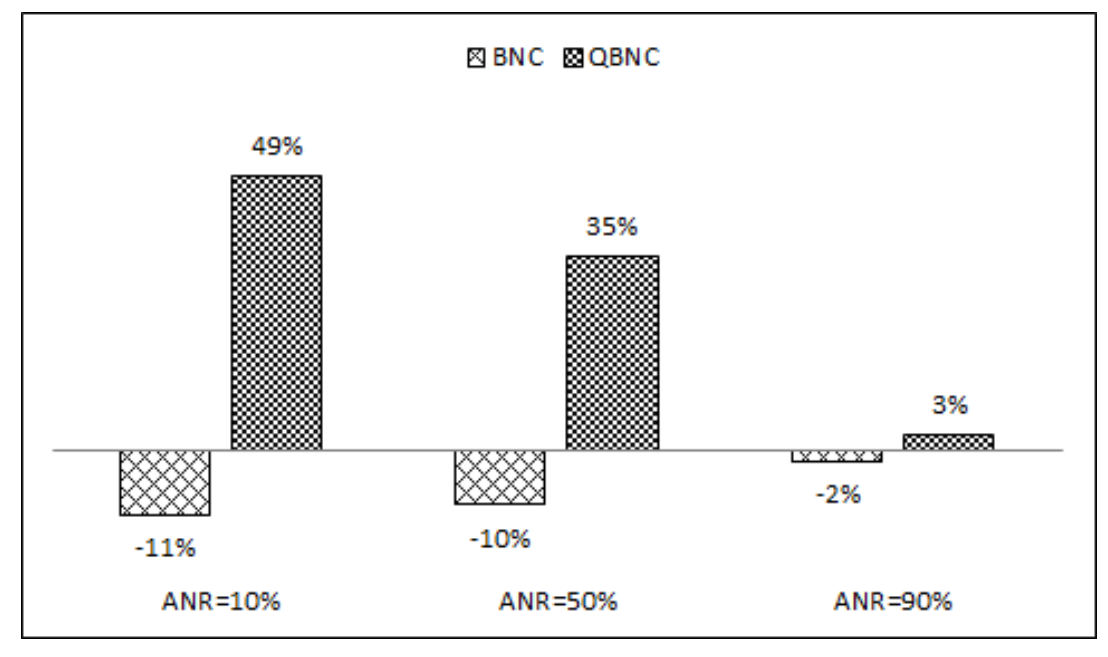

Figure 10. The RTOC improvement on network throughput.

Table 3 summarizes the relative performance of RTOC in terms of PDR, end-to-end 
delay, packet inter-arrival jitter, throughput, and COR. The results show that the QBNC MAC mode improves the performance of RTOC in terms of all metrics, while the BNC MAC mode slightly degrades the PDR, jitter, and throughput. However, BNC gives better improvement in terms of end-to-end delay. The results also show that the number of active nodes has a direct impact on the performance of RTOC. In specific, increasing ANR has a negative impact on the QBNC improvement. On the other hand, this impact is barely noticeable on BNC.

Table 3. RTOC improvement on the performance of RTP using the BNC and QBNC MAC modes compared to the WNC mode for different active node ratios ( $\mathrm{ANR}=10 \%, 50 \%$, and $90 \%$ ).

\begin{tabular}{|c|c|c|c|c|c|c|}
\hline \multirow{2}{*}{ Performance Metrics } & \multicolumn{2}{|c|}{$A N R=10 \%$} & \multicolumn{2}{|c|}{$\mathrm{ANR}=\mathbf{5 0} \%$} & \multicolumn{2}{|c|}{$\mathrm{ANR}=90 \%$} \\
\hline & $B N C$ & $Q B N C$ & $B N C$ & $Q B N C$ & $B N C$ & $Q B N C$ \\
\hline Packet Delivery Ratio (PDR) & $-3 \%$ & $33 \%$ & $-4 \%$ & $24 \%$ & $-8 \%$ & $9 \%$ \\
\hline End-to-End Packet Delay & $57 \%$ & $38 \%$ & $48 \%$ & $30 \%$ & $50 \%$ & $21 \%$ \\
\hline Packet Inter-Arrival Jitter & $-13 \%$ & $33 \%$ & $-10 \%$ & $26 \%$ & $-2 \%$ & $3 \%$ \\
\hline Network Throughput & $-11 \%$ & $49 \%$ & $-10 \%$ & $35 \%$ & $-2 \%$ & $3 \%$ \\
\hline Coding Opportunity Ratio (COR) & $12 \%$ & $27 \%$ & $12 \%$ & $18 \%$ & $9 \%$ & $13 \%$ \\
\hline
\end{tabular}

In summary, the results illustrate that RTOC has a decent potential to improving the performance of real-time multimedia transmission. In specific, RTOC has shown to improve the overall performance in terms of packet delivery ratio, end-to-end delay, packet inter-arrival jitter, and throughput, especially when the QBNC MAC mode is used.

\section{Conclusion}

This paper introduces RTOC, a new XOR-based opportunistic network coding architecture that copes with the performance characteristics required for real-time transmission. RTOC provides an efficient framework for applying various techniques to satisfy different multimedia application requirements such as bandwidth, delay, delay variation and loss. The simulation results demonstrated that RTOC has a good potential to improve the performance metrics of real-time multimedia transmission such as packet delivery ratio, end-to-end delay, jitter, and throughput. As a future work, the impact of the various network conditions and protocols used, such as the routing and the channel access algorithms, will be investigated.

\section{References}

[1] Krasic C., Li K., Walpole J., "The case for streaming multimedia with TCP”, The 8th International Workshop on Interactive Distributed multimedia systems (iDMS 2001). Lancaster, UK, September 2001. http://dx.doi.org/10.1007/3-540-44763-6_22

[2] Postel J., "User Datagram Protocol”, RFC 768, Internet Engineering Task Force, 1980.

[3] Schulzrinne H., Casner S., Frederick R., Jacobson V., "RTP: a Transport Protocol for Real-time Applications", RFC 3550, Internet Engineering Task Force, 2003.

[4] Ahlswede R., Cai N. C. N., Li S. Y. R., Yeung R. W., "Network Information Flow". IEEE 
Transactions on Information Theory. Vol. 46, No. 4, pp. 1204-1216, 2000. http://dx.doi.org/10.1109/18.850663

[5] Fragouli C., Katabi D., Markopoulou A., Medard M., Rahul H., "Wireless network coding: opportunities \& challenges", IEEE Military Communications Conference (MILCOM 2007). Orlando, Florida, October 2007. http://dx.doi.org/10.1109/MILCOM.2007.4454988

[6] Ramamoorthy A., Shi J., Wesel R. D., "On the Capacity of Network Coding for Random Networks". IEEE Transactions on Information Theory. Vol. 51, No. 8, pp. 2878-2885, 2005. http://dx.doi.org/10.1109/TIT.2005.851725

[7] Lun D. S., Ramakar N., Koetter R., Medard M., Ahmed E., "Achieving minimum-cost multicast: a decentralized approach based on network coding", 24th Annual Joint Conference of the IEEE Computer and Communications Societies (INFOCOM 2005). Miami, Florida, March 2005. http://dx.doi.org/10.1109/INFCOM.2005.1498443

[8] Katti S., Katabi D., Hu W., Rahul H., Medard M., "The importance of being opportunistic: practical network coding for wireless environments," The 43rd Annual Allerton Conference on Communication, Control and Computing. Monticello, Illinois, September 2005.

[9] Fragouli C., Widmer J., Le Boudec J. Y., "Energy-efficient broadcasting in wireless ad-hoc networks using network coding", The First Workshop on Network Coding (NetCod 2005). Riva del Garda, Italy, April 2005.

[10] Fragouli C., Widmer J., Le Boudec J. Y., "A network coding approach to energy efficient broadcasting: from theory to practice", 25th IEEE International Conference on Computer Communications (INFOCOM 2006). Barcelona, Spain, April 2006. http://dx.doi.org/10.1109/INFOCOM.2006.45

[11] Widmer J., Le Boudec J. Y., "Network coding for efficient communication in extreme networks," The 2005 ACM SIGCOMM Workshop on Delay-tolerant Networking (WDTN 05). Philadelphia, PA, August 2005. http://dx.doi.org/10.1145/1080139.1080147

[12] Dimakis G., Prabhakaran V., Ramchandran K., "Ubiquitous access to distributed data in large-scale sensor networks through decentralized erasure codes", The Fourth International Symposium on Information Processing in Sensor Networks 2005 (IPSN 2005). Los Angeles, California, April 2005. http://dx.doi.org/10.1109/IPSN.2005.1440909

[13] Zhang S., Lam P. P., "Hot topic: physical-layer network coding", The 12th Annual International Conference on Mobile Computing and Networking (MOBICOM '06). Los Angeles, California, September 2006. http://dx.doi.org/10.1145/1161089.1161129

[14] Katti S., Gollakota S., Katabi D., "Embracing wireless interference: analog network coding", The 2007 conference on Applications, Technologies, Architectures, and Protocols for Computer Communications (SIGCOMM 2007). Kyoto, Japan, 2007. http://dx.doi.org/10.1145/1282380.1282425

[15] Katti S., Katabi D., "MIXIT: the network meets the wireless channel", The Sixth ACM Workshop on Hot Topics in Networks (HotNets-VI). 2006.

[16] Dimakis G., Godfrey P. B., Wu Y., Wainwright M. J., Ramchandran K., "Network coding for distributed storage systems", The 26th IEEE International Conference on Computer Communications (INFOCOM 2007). Anchorage, Alaska, May 2007. http://dx.doi.org/10.1109/INFCOM.2007.232 
[17] Wu Y., Chou P. A., Kung S. Y., "Information exchange in wireless networks with network coding and physical-layer broadcast", Microsoft, Technical Report, MSR-TR-2004-78, 2004.

[18] Katti S., Rahul H., Katabi D., Medard M., Crowcroft J., "XORs in the Air: Practical Wireless Network Coding”. IEEE/ACM Transactions on Networking. Vol. 16, No. 3, pp. 497-510, 2008. http://dx.doi.org/10.1109/TNET.2008.923722

[19] Chachulski S., Jennings M., Katti S., Katabi D., "Trading structure for randomness in wireless opportunistic routing", The 2007 Conference on Applications, Technologies, Architectures, and Protocols for Computer Communications (SIGCOMM 2007). Kyoto, Japan, 2007. http://dx.doi.org/10.1145/1282380.1282400

[20] Katti S., Katabi D., Balakrishnan H., Medard M., "Symbol-level network coding for wireless mesh networks", The ACM SIGCOMM 2008 Conference on Data Communication (SIGCOMM 2008). Seattle, WA, August 2008. http://dx.doi.org/10.1145/1402958.1403004

[21] Radunovic B., Gkantsidis C., Key P., Rodriguez P., Hu W., "An optimization framework for practical multi-path routing in wireless mesh networks", Microsoft, Technical Report, MSR-TR-2007-81, 2007.

[22] Nguyen D., Nguyen T., Yang X., "Multimedia wireless transmission with network coding", The 16th International Packet Video Workshop (Packet Video 2007). Lausanne, Switzerland, November 2007. http://dx.doi.org/10.1109/PACKET.2007.4397057

[23] Nguyen D., Nguyen T., "Network coding-based wireless media transmission using POMDP", The 17th International Packet Video Workshop. Seattle, WA, June 2009. http://dx.doi.org/10.1109/PACKET.2009.5152148

[24] Seferoglu H., Markopoulou A., "Opportunistic network coding for video streaming over wireless", The 16th International Packet Video Workshop (Packet Video 2007). Lausanne, Switzerland, November 2007. http://dx.doi.org/10.1109/PACKET.2007.4397041

[25] Seferoglu H., Markopoulou A., "Video-Aware Opportunistic Network Coding over Wireless Networks". IEEE Journal on Selected Areas in Communications. Vol. 27, No. 5, pp. 713-728, 2009. http://dx.doi.org/10.1109/JSAC.2009.090612

[26] Nguyen D., Nguyen T., Yang X., "Joint Network Coding and Scheduling for Media Streaming over Multiuser Wireless Networks". IEEE Transactions on Vehicular Technology. Vol. 60, No. 3, pp. 1086 -1098, 2011. http://dx.doi.org/10.1109/TVT.2011.2112677

[27] Wang H., Chang R. Y., Kuo C. C. J., "Wireless multi-party video conferencing with network coding", The 2009 IEEE International Conference on Multimedia and Expo (ICME 2009). New York, NY, June/July 2009. http://dx.doi.org/10.1109/ICME.2009.5202786

[28] Seferoglu H., Markopoulou A., "Delay-optimized network coding for video streaming over wireless networks", The 2010 IEEE International Conference on Communications (ICC 2010). Cape Town, South Africa, May 2010. http://dx.doi.org/10.1109/ICC.2010.5502651

[29] Nguyen D., Tran T., Nguyen T., Bose B., "Wireless Broadcast Using Network Coding". IEEE Transactions on Vehicular Technology. Vol. 58, No. 2, pp. 914-925, 2009. http://dx.doi.org/10.1109/TVT.2008.927729

[30] Zhan C., Xu Y., Wang J., Lee V. C. S., "Reliable multicast in wireless networks using network coding", The IEEE 6th International Conference on Mobile Adhoc and Sensor Systems 2009). Macau, China, October 2009. 
http://dx.doi.org/10.1109/MOBHOC.2009.5336960

[31] Rayanchu S. K., Sen S., Wu J., Banerjee S., Sengupta S., "Loss-aware network coding for unicast wireless sessions: design, implementation, and performance evaluation", The 2008 ACM SIGMETRICS International Conference on Measurement and Modeling of Computer Systems (SIGMETRICS 2008). Annapolis, MD, USA, 2008. http://dx.doi.org/10.1145/1375457.1375468

[32] Cui T., Chen L., Ho T., "Energy efficient opportunistic network coding for wireless networks", The 27th Conference on Computer Communications (INFOCOM 2008). Phoenix, AZ, USA, 2008. http://dx.doi.org/10.1109/INFOCOM.2008.81

[33] Seferoglu H., Markopoulou A., Medard M., "NCAPQ: Network coding-aware priority queueing for UDP flows over COPE", 2011 International Symposium on Network Coding (NetCode), Beijing, China, July 2011. http://dx.doi.org/10.1109/ISNETCOD.2011.5979091

[34] Chen W., Letaief K. B., Cao Z., "Opportunistic network coding for wireless networks", The 2007 IEEE International Conference on Communications (ICC 2007). Glasgow, Scotland, UK, June 2007. http://dx.doi.org/10.1109/ICC.2007.765

[35] Varga A., Hornig, R., "An overview of the OMNeT++ simulation environment", The 1st International Conference on Simulation Tools and Techniques for Communications, Networks and Systems. Marseille, France, March 2008. http://dx.doi.org/10.4108/ICST.SIMUTOOLS2008.3027

[36] “INET Framework for OMNeT++," [Online]. Available at: http://inet.omnetpp.org [37] "INETMANET Framework for OMNeT++ based on INET Framework," [Online]. Available at: https://github.com/inetmanet/inetmanet/wiki

[38] Hoffman D., Fernando G., Goyal V., Civanlar M., "RTP Payload Format for MPEG1/MPEG2 Video," RFC 2250, 1998.

\section{Copyright Disclaimer}

Copyright reserved by the author(s).

This article is an open-access article distributed under the terms and conditions of the Creative Commons Attribution license (http://creativecommons.org/licenses/by/3.0/). 